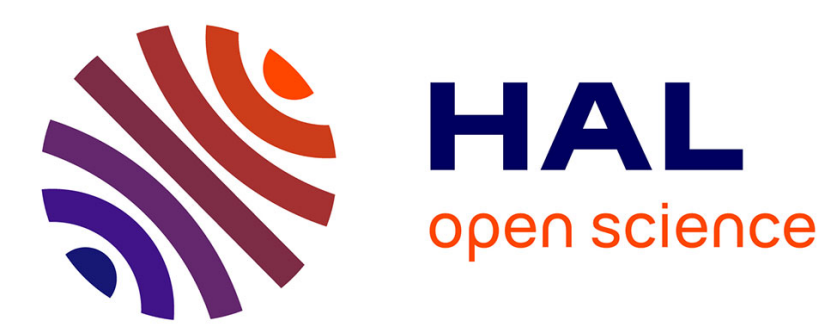

\title{
Numerical studies of porous ductile materials containing arbitrary ellipsoidal voids - I: Yield surfaces of representative cells
}

Komlanvi Madou, Jean-Baptiste Leblond

\section{- To cite this version:}

Komlanvi Madou, Jean-Baptiste Leblond. Numerical studies of porous ductile materials containing arbitrary ellipsoidal voids - I: Yield surfaces of representative cells. European Journal of Mechanics A/Solids, 2013, 42, pp.480 - 489. 10.1016/j.euromechsol.2013.06.004 . hal-01436280

\section{HAL Id: hal-01436280 \\ https: / hal.sorbonne-universite.fr/hal-01436280}

Submitted on 16 Jan 2017

HAL is a multi-disciplinary open access archive for the deposit and dissemination of scientific research documents, whether they are published or not. The documents may come from teaching and research institutions in France or abroad, or from public or private research centers.
L'archive ouverte pluridisciplinaire HAL, est destinée au dépôt et à la diffusion de documents scientifiques de niveau recherche, publiés ou non, émanant des établissements d'enseignement et de recherche français ou étrangers, des laboratoires publics ou privés. 


\title{
Numerical studies of porous ductile materials containing arbitrary ellipsoidal voids - I: Yield surfaces of representative cells
}

\author{
Komlanvi Madou, Jean-Baptiste Leblond* \\ UPMC Univ Paris 6 and CNRS, UMR 7190, Institut Jean Le Rond d'Alembert, F-75005 \\ Paris, France
}

\begin{abstract}
This work is devoted to some numerical limit-analyses, performed by the finite element method, of elementary cells of arbitrary ellipsoidal shape and containing confocal ellipsoidal voids. The aim is essentially, in the present Part I, to validate an approximate analytic criterion for such cells recently proposed by Madou and Leblond (2012a,b), and in Part II, to supplement the model by proposing reasonable evolution equations for the length and orientation of the axes of the voids. The results obtained are however not specifically attached to this particular model and could assist in the definition of any similar one for plastic porous materials incorporating void shape effects.

The present Part I is devoted to the numerical determination of the yield surfaces corresponding to eight different void geometries (prolate and oblate spheroids, circular and elliptic cylinders, circular and elliptic cracks, two general ellipsoids). The results obtained confirm the general validity of Madou and Leblond (2012a,b)'s proposed criterion, although slight corners not accounted for in this criterion are apparent on the numerical yield surfaces of cylindrical cells.
\end{abstract}

Keywords: Porous ductile solids; ellipsoidal voids; numerical study; overall yield surface

\section{Introduction}

Most models of the homogenized behavior of porous ductile solids were derived through limit-analysis of hollow "representative cells" loaded through conditions of homogeneous boundary strain rate (Mandel, 1964; Hill, 1967). The first and most classical model of this type was defined by Gurson (1977). This model applied to spherical voids only and therefore disregarded void shape effects. In order to account for such effects, Gologanu et al. (1993, 1994); Gologanu (1997); Gologanu et al. (1997) extended Gurson (1977)'s work to both prolate and oblate spheroidal (axisymmetric ellipsoidal) voids; their model is currently referred to as the GLD model.

\footnotetext{
* Corresponding author.
} 
The approach initiated by Gurson (1977) and Gologanu et al. (1993, 1994); Gologanu (1997); Gologanu et al. (1997) was pursued by many authors; see the recent synthesis of Benzerga and Leblond (2010) on this topic and the references included therein. But although Gurson's model and the GLD model were extended in various directions, no extension to general (non-spheroidal) ellipsoidal voids was envisaged for a long time, although such voids do occur in practical situations, notably in laminated plates, see e.g. Table 4 in (Benzerga et al., 1999) and Table 2 in (Benzerga et al., 2004).

Such an extension was however envisaged in a very recent work of Madou and Leblond (2012a,b). In order to define an approximate homogenized criterion for plastic porous materials containing voids of arbitrary ellipsoidal shape, these authors followed the approach initiated by Gurson (1977) and Gologanu et al. (1993, 1994); Gologanu (1997); Gologanu et al. (1997), but also introduced new elements. Their "hybrid" method of derivation indeed combined (i) analytical limit-analysis of elementary hollow ellipsoidal cells loaded through conditions of homogeneous boundary strain rate, based on a family of velocity fields recently discovered by Leblond and Gologanu (2008); (ii) rigorous bounds for nonlinear composites derived by Ponte-Castaneda (1991), Willis (1991) and Michel and Suquet (1992) using previous results of Willis (1977); and (iii) micromechanical finite element simulations.

It is important to note, however, that the problem of general ellipsoidal voids was envisaged as early as 1994 by Ponte-Castaneda and coworkers, following a completely different line of thought based on the concept of "linear comparison material". The early model of Ponte-Castaneda and Zaidman (1994), in spite of its accuracy for deviatoric loadings, suffered from a notable overestimation of the yield limit under hydrostatic loading. This drawback was remedied in the more recent model of Danas and Ponte-Castaneda (2009a,b) based on Ponte-Castaneda (2002a)'s "second-order homogenization method"; however micromechanical numerical simulations remain to be performed to assess the accuracy of the approximate yield surface proposed. Ponte-Castaneda and Zaidman (1994)'s yield surface was also very recently improved by Agoras and Ponte-Castaneda (2013) using another approach, and the new yield surface obtained was shown to agree very well with that of Madou and Leblond (2012a,b) - provided that the "distribution function" of the centers of the voids was assumed to be identical to that postulated by the latter authors. Thus the model of Madou and Leblond (2012a,b) and those recently proposed by Ponte-Castaneda and coworkers for general ellipsoidal voids represent possible alternatives, the advantage of the former model being its greater formal simplicity, and the advantage of the others their stronger degree of internal coherence. ${ }^{1}$

At this stage of development of Madou and Leblond (2012a,b)'s model, however, two elements are still lacking:

- a validation of the criterion proposed, based on numerical simulations of various hollow cells of the type considered in its derivation;

- some suitable evolution equations for the length and orientation of the axes of the voids, which act as internal parameters in the model.

$\overline{1}$ The "hybrid" character of Madou and Leblond (2012a,b)'s model has been noted above. 
The aim of the present work is to fill these two gaps, in Parts I and II respectively.

It is important to note that although our attention is focussed here on Madou and Leblond (2012a,b)'s model, the collection of numerical yield surfaces of hollow ellipsoidal cells that will be determined in the present Part I will potentially be usable for the validation of any similar yield criterion for plastic porous materials incorporating void shape effects (assuming of course that the hypotheses made on the geometry of the cells are the same).

The present paper is organized as follows:

- Section 2 presents the principle of the numerical simulations. The equations solved are those of limit-analysis neglecting elastic strains and considering only plastic ones. (This is almost always a sound approximation in the context of ductile rupture). Although the use of special finite element techniques disregarding elasticity (Pastor, 1978; de Buhan and Maghous, 1995; Pastor et al., 2009) would be possible here, we employ instead a standard finite element code including elasticity, the use of a single sufficiently large load step ensuring the practical equivalence of the time-discretized finite element equations and those of limit-analysis.

- Section 3 then displays the numerical yield surfaces obtained in eight different cases covering a wide variety of void geometries: prolate and oblate spheroids, circular and elliptic cylinders, circular and elliptic cracks, and two general ellipsoids. The formidable task of numerical determination of the entire yield locus, that is a 5D hypersurface in the $6 \mathrm{D}$ space of overall stress tensors, is reduced to reasonable proportions by (i) considering only stress tensors having the same principal axes as the voids, thereby reducing the dimension of the stress space to 3 and that of the yield locus to 2; and (ii) determining only the traces of this yield locus in three distinct planes. A few results concerning the normal to the yield locus, which determines the direction of overall plastic flow, are also presented.

\section{Principle of numerical calculations}

\subsection{Generalities}

Numerical limit-analysis calculations are performed using the standard plasticity option (including elasticity) of the commercial SYSTUS ${ }^{\circledR}$ finite element code developed by ESI Group. Instead of simulating the gradual loading of the structure, we impose a single, large load step so as to immediately reach the limit-load (without however updating the geometry since limit-analysis disregards geometric changes). This procedure does not introduce any error of principle since as shown in Appendix A, under such conditions, the equations of the time-discretized finite element problem are exactly equivalent to those of limit-analysis, provided that an implicit algorithm is used for the projection of the elastic stress predictor onto the yield locus.

The SYSTUS ${ }^{\circledR}$ code offers the possibility of writing higher level programmes managing and exploiting calculations; three such programmes have been developed: 
- The first prepares the mesh. Starting from a hollow sphere or circular cylinder meshed with standard means, it deforms the geometry into an ellipsoid or an elliptic cylinder containing an ellipsoidal or cylindrical confocal void (as envisaged in Madou and Leblond (2012a,b)'s model).

- The second programme runs the calculations. Its main task is to adjust the components of the overall strain imposed by some Newton method, so as match the desired values of the ratios of the overall stress components.

- The third programme exploits the results by calculating the components of the overall stress, the average strain of the void and in some cases its average rotation.

In all calculations the values of the material constants are as follows: Young's modulus, $E=210,000 \mathrm{MPa}$; Poisson's ratio, $\nu=0.499 ;{ }^{2}$ yield stress in simple tension, $\sigma_{0}=$ $300 \mathrm{MPa}$. A BFGS method is used for the global elastoplastic iterations; a few hundred iterations are typically necessary for satisfactory convergence.

In all cases, the major, intermediate and minor semi-axes of the void are denoted $a, b$ and $c$, the corresponding directions $x, y$ and $z$, and the porosity (void volume fraction) $f$.

\subsection{Ellipsoidal geometries}

Most cells considered are general ellipsoids containing a confocal ellipsoidal void, subjected to conditions of homogeneous boundary strain (Mandel, 1964; Hill, 1967):

$$
\mathbf{u}(\mathbf{X})=\mathbf{E} \cdot \mathbf{X} \quad \text { on } \partial \Omega
$$

where $\mathbf{u}$ denotes the displacement, $\mathbf{X}$ the initial position-vector, $\mathbf{E}$ the overall strain (symmetric second-rank tensor) imposed and $\partial \Omega$ the external boundary of the cell $\Omega$. For these geometries we consider only tensors $\mathbf{E}$ having principal directions identical to those of the void and the cell; this implies existence of symmetries about three perpendicular planes permitting to mesh only $1 / 8$ of the structure.

The mesh consists of 26,481 nodes and 24,000 trilinear selectively subintegrated 8-node brick elements; there are 20 elements in the radial direction and 40 elements in the two perpendicular directions. Figure 1 shows the mesh of a typical cell having $(a, b, c)=$ $(10,5,1)$ and $f=0.01$.

The overall stress tensor $\boldsymbol{\Sigma}$ is evaluated from its local counterpart $\boldsymbol{\sigma}$ through the formula

$$
\boldsymbol{\Sigma}=\frac{1}{\operatorname{vol}(\Omega)} \int_{\Omega-\omega} \boldsymbol{\sigma} d V
$$

where $\omega$ represents the void and thus $\Omega-\omega$ the sound domain.

\footnotetext{
$\overline{2}$ This value, which ensures approximate elastic incompressibility of the material, is acceptable since limit-loads are independent of elastic constants, and facilitates convergence of the global iterations since the displacement field resulting from the first, purely elastic iteration already satisfies the necessary incompressibility condition.
} 


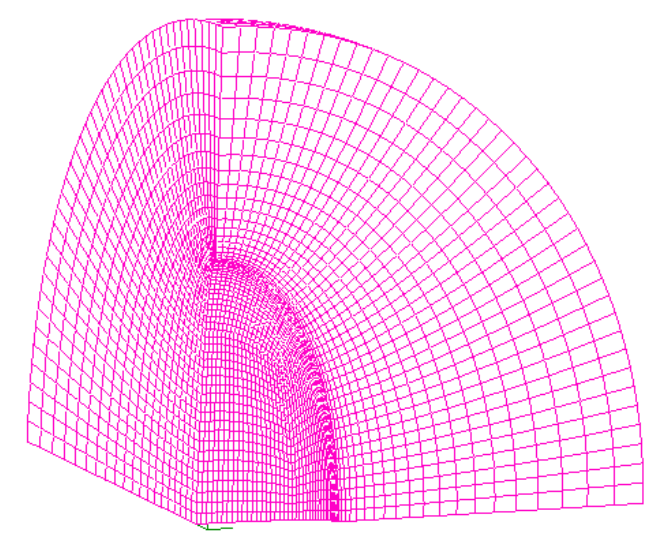

Fig. 1. Mesh of a general ellipsoidal cell - $(a, b, c)=(10,5,1), f=0.01$

\subsection{Cylindrical geometries}

We also consider, as special cases, circular or elliptic cylindrical cells containing a confocal cylindrical void. For such geometries, thanks to translational invariance in the direction $x$ of the axis, only one layer of material need be meshed. One must therefore distinguish between the cell itself, which is an infinitely long cylinder, and the layer meshed, $\Omega$.

The external lateral surface of the layer $(\partial \Omega)^{\mathrm{L}}$, being a part of the boundary of the cell, is subjected to conditions of homogeneous boundary strain like in the case of ellipsoidal cells:

$$
\mathbf{u}(\mathbf{X})=\mathbf{E} . \mathbf{X} \quad \text { on }(\partial \Omega)^{\mathrm{L}},
$$

but the tensor $\mathbf{E}$ is allowed here to be non-diagonal.

On the other hand, the top surface of the layer $(\partial \Omega)^{\mathrm{T}}$ and its bottom one $(\partial \Omega)^{\mathrm{B}}$ must not be subjected to such conditions since they are not parts of the cell boundary. Because of the obvious periodicity in the direction $x$, the conditions to be imposed on them are:

$$
\mathbf{u}(\mathbf{X})=\mathbf{E} \cdot \mathbf{X}+\widetilde{\mathbf{u}}(\mathbf{X}) \quad \text { on }(\partial \Omega)^{\mathrm{T}} \cup(\partial \Omega)^{\mathrm{B}}
$$

where $\widetilde{\mathbf{u}}(\mathbf{X})$ is some unknown periodic displacement field. Eliminating this field by taking the difference between the conditions imposed on $(\partial \Omega)^{\mathrm{T}}$ and $(\partial \Omega)^{\mathrm{B}}$, one gets the condition

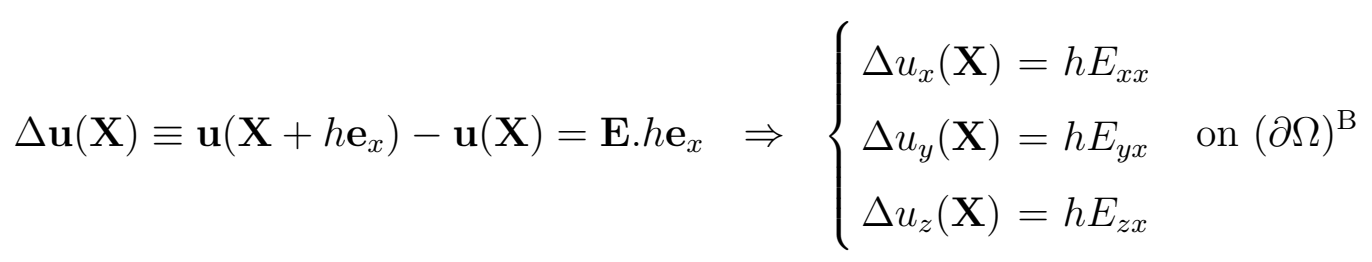

where $h$ denotes the thickness of the layer and $\mathbf{e}_{x}$ the unit vector parallel to the direction $x$.

The meshes consists of 9,920 nodes and 4,800 trilinear selectively subintegrated 8-node brick elements; there are 30 elements in the radial direction and 160 elements in the orthoradial direction. Figure 2 shows a typical mesh having $(a, b, c)=(+\infty, 5,5)$ and 
$f=0.01$. Conditions (5) are imposed by connecting the top and bottom surfaces through special, very stiff $1 \mathrm{D}$ elements.

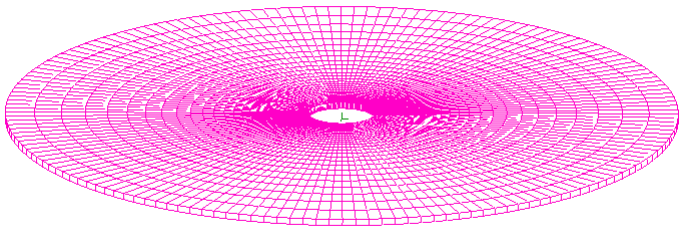

Fig. 2. Meshes of a circular cylindrical cell - $(a, b, c)=(+\infty, 5,5), f=0.01$

The overall stress tensor $\boldsymbol{\Sigma}$ is evaluated using the same formula (2) as before.

\section{Yield surfaces of hollow ellipsoidal cells}

For the determination of numerical yield loci, we consider only overall strain tensors having the same principal directions as the ellipsoidal cell; the same is then true of the overall stress tensor, which thus possesses three independent components only, $\Sigma_{x x}, \Sigma_{y y}$ and $\Sigma_{z z}$. The reason for considering only such stress states is that in Madou and Leblond (2012a,b)'s model, the yield limits under shear stresses $\Sigma_{x y}, \Sigma_{y z}, \Sigma_{z x}$ are deduced from the nonlinear Willis bound - as originally established by Willis (1977) in the elastic case and extended by Ponte-Castaneda (1991), Willis (1991) and Michel and Suquet (1992) to the plastic case - which is known to be quite accurate for such loadings; thus a validation of these yield limits is not deemed necessary. Also, consideration of non-diagonal stress states would destroy symmetries and require more complete meshes, thus making the calculations extremely heavy, at least for general ellipsoidal geometries.

Even so, the yield locus is still a 2D surface in a 3D space, the full determination of which would be difficult. We therefore determine only the traces of this surface in the three planes of respective equations $\Sigma_{x x}=\Sigma_{y y}, \Sigma_{x x}=\Sigma_{z z}, \Sigma_{y y}=\Sigma_{z z}$. (Note that all planes contain the "hydrostatic line" $\Sigma_{x x}=\Sigma_{y y}=\Sigma_{z z}$ ). The obvious invariance of the yield locus in the transformation $\Sigma \rightarrow-\Sigma$ also permits to plot only half of these traces.

An important remark is that by a well-known theorem of limit-analysis, the direction of plastic flow is identical to that of the normal to the yield locus, so that a study of this normal is also of some interest. However, with regard to this topic, we shall not present a systematic study but be content with a selection of results for space reasons.

\subsection{Prolate spheroidal void}

Figure 3 shows the results obtained for the yield locus of a prolate spheroidal void having $(a, b, c)=(5,1,1)$ and $f=0.01$. The quantities plotted on the horizontal and vertical axes are the overall mean stress $\left(\Sigma_{m} \equiv \frac{1}{3} \operatorname{tr} \boldsymbol{\Sigma}\right)$ and deviatoric stress $\left(\Sigma_{x x}-\Sigma_{z z}\right)$, "normalized" 
by the yield stress $\sigma_{0}$ in simple tension. Because of rotational symmetry about the direction $x$, the traces of the yield locus in the planes $\Sigma_{x x}=\Sigma_{y y}$ and $\Sigma_{x x}=\Sigma_{z z}$ are identical, so only the first one is represented. In Figure 3(a) for axisymmetric loadings $\left(\Sigma_{y y}=\Sigma_{z z}\right)$, we plot, in addition to the finite element results (Num) and the approximate predictions of the Madou-Leblond model (Model), the former numerical results of Gologanu et al. (1993); Gologanu (1997); Gologanu et al. (1997) (Num GLD) and the approximate predictions of the GLD model (Model GLD); this makes sense since these earlier numerical results and approximate predictions were also for axisymmetric loadings. In Figure 3(b) for non-axisymmetric loadings having $\Sigma_{x x}=\Sigma_{y y}$, the sole present numerical results and approximate predictions of the Madou-Leblond model are represented.

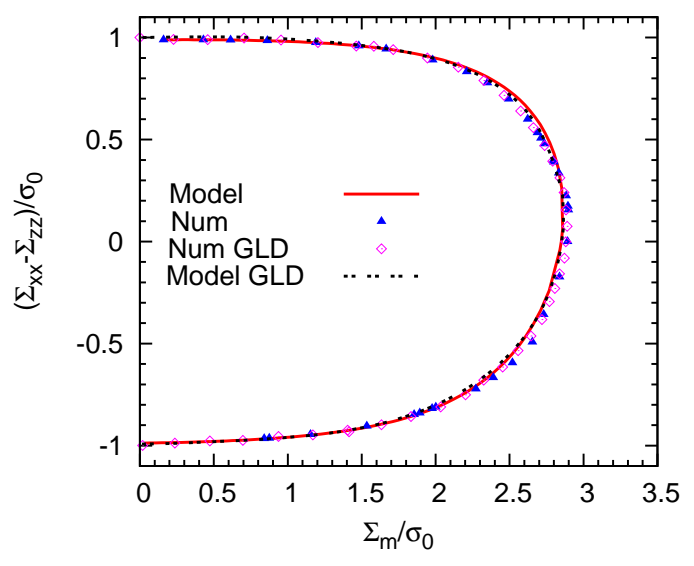

(a) $\Sigma_{y y}=\Sigma_{z z} \neq \Sigma_{x x}$

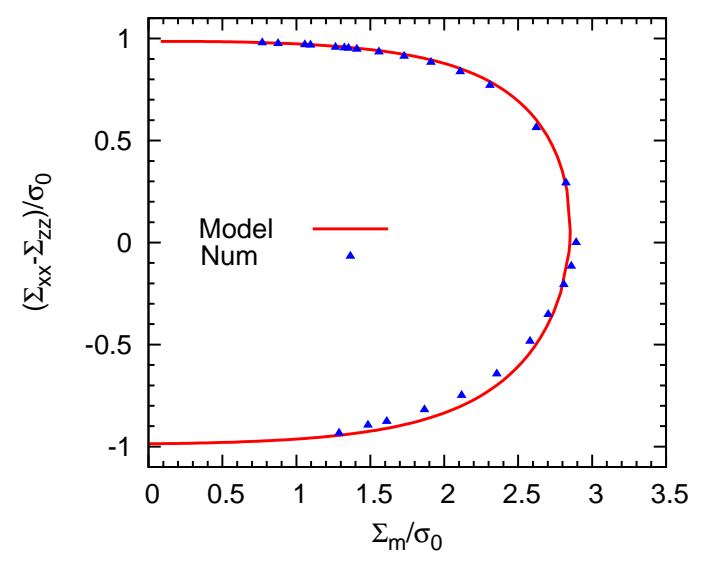

(b) $\Sigma_{x x}=\Sigma_{y y} \neq \Sigma_{z z}$

Fig. 3. Traces in two planes of the yield locus for a prolate void - $(a, b, c)=(5,1,1), f=0.01$ Numerical results and model predictions (Madou-Leblond, GLD)

The excellent agreement between the present numerical results and those obtained by Gologanu et al. (1993); Gologanu (1997); Gologanu et al. (1997) by a completely different method (minimization of the overall plastic dissipation over a large class of analytical trial velocity fields proposed by Lee and Mear (1992)) is a strong argument in favor of the correctness and accuracy of all of them. Observe also that both the Madou-Leblond model and the GLD model (for axisymmetric loadings) make a very good job of reproducing the numerical results, in spite of the different expressions of the various coefficients involved in the approximate yield functions proposed.

Figure 4 provides a partial representation of the direction of the normal to the yield surface for (non-axisymmetric) loadings having $\Sigma_{x x}=\Sigma_{y y}$. The quantity represented is the ratio of the $y y$ and $x x$ components of the theoretical overall strain rate $\mathbf{D}$, identified to the ratio of the same components of the numerical overall strain $\mathbf{E}$, using the equivalence explained in Appendix A between the equations of limit-analysis and those of the finite element problem. It is plotted versus the quantity $\arctan \left(\frac{\Sigma_{x x}-\Sigma_{z z}}{\Sigma_{m}}\right)$ measuring position along the trace of the yield surface in the plane $\Sigma_{x x}=\Sigma_{y y}$. It may be noted that for a spherical void and the loadings envisaged, one would have $D_{x x}=D_{y y}$ for symmetry reasons; thus the deviations of the ratio $D_{y y} / D_{x x}$ from unity are a consequence of non-sphericity, and their nice reproduction by the Madou-Leblond model illustrates the correct incorporation of void shape effects in this model. 


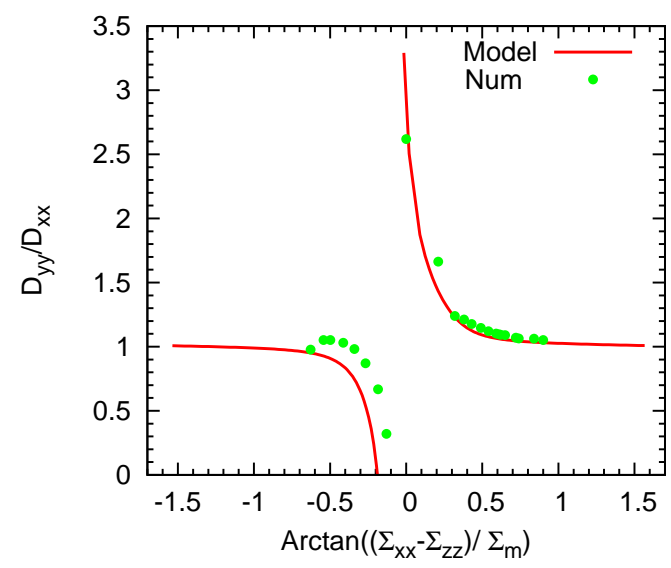

Fig. 4. Direction of plastic flow for a prolate void - $(a, b, c)=(5,1,1), f=0.01, \Sigma_{x x}=\Sigma_{y y} \neq \Sigma_{z z}$ - Numerical results and model predictions (Madou-Leblond)

\subsection{Oblate spheroidal void}

Figure 5 shows results for the yield locus of an oblate spheroidal void having $(a, b, c)=$ $(5,5,1)$ and $f=0.01$. Again, Figure $5(\mathrm{a})$ is for axisymmetric loadings $\left(\Sigma_{x x}=\Sigma_{y y}\right)$, for which former results of Gologanu et al. (1994); Gologanu (1997); Gologanu et al. (1997) are available and represented, and Figure 5(b) is for non-axisymmetric loadings $\left(\Sigma_{x x}=\Sigma_{z z}\right)$. Exactly the same remarks as in the case of a prolate spheroidal void may be made here.

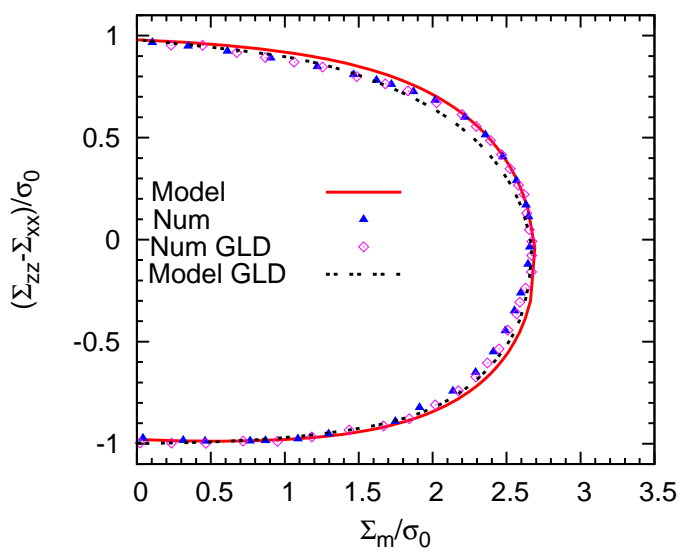

(a) $\Sigma_{x x}=\Sigma_{y y} \neq \Sigma_{z z}$

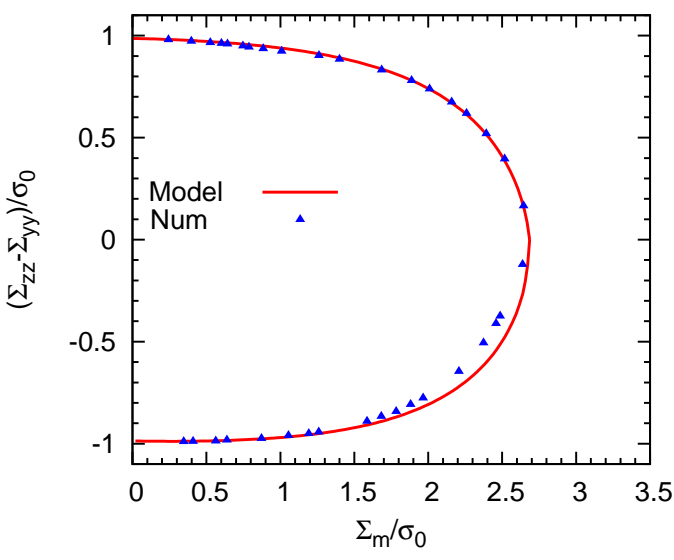

(b) $\Sigma_{x x}=\Sigma_{z z} \neq \Sigma_{y y}$

Fig. 5. Traces in two planes of the yield locus for an oblate void - $(a, b, c)=(5,5,1), f=0.01$ Numerical results and model predictions (Madou-Leblond, GLD)

Figure 6 provides a partial representation of the direction of the normal to the yield surface for (non-axisymmetric) loadings having $\Sigma_{x x}=\Sigma_{z z}$. The quantity represented is the ratio $D_{z z} / D_{x x}$, plotted versus the quantity $\arctan \left(\frac{\Sigma_{z z}-\Sigma_{y y}}{\Sigma_{m}}\right)$. Again, for a spherical void and the loadings envisaged, one would have $D_{x x}=D_{z z}$ for symmetry reasons, so that the deviations of the ratio $D_{z z} / D_{x x}$ from unity are a consequence of non-sphericity. The Madou-Leblond model provides an acceptable representation of the numerical results, which again shows that this model correctly incorporates void shape effects; but the greater gap than in the prolate case between the numerical and theoretical yield loci near 
the "hydrostatic axis" (compare Figures 3(b) and 5(b)) makes the reproduction of the numerical normal by the model less accurate in this region.

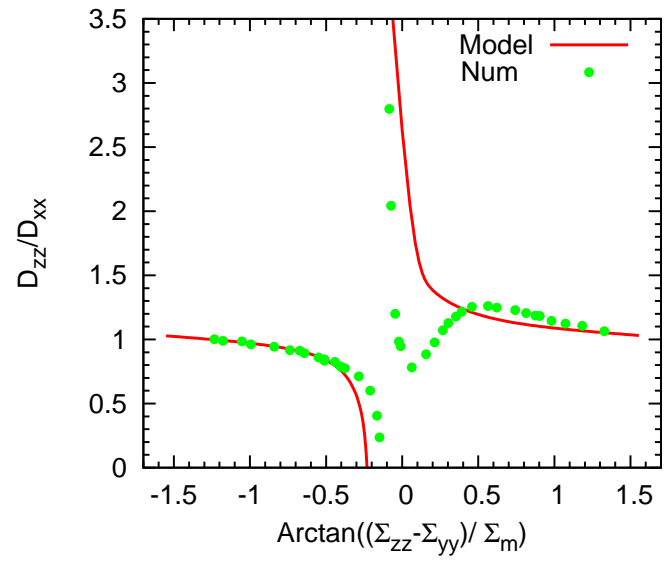

Fig. 6. Direction of plastic flow for an oblate void - $(a, b, c)=(5,5,1), f=0.01, \Sigma_{x x}=\Sigma_{z z} \neq \Sigma_{y y}$ - Numerical results and model predictions (Madou-Leblond)

\subsection{Circular cylindrical void}

Figure 7 is for a circular cylindrical void (extreme case of a prolate spheroidal void) having $(a, b, c)=(+\infty, 5,5)$ and $f=0.01$. Again, Figure $7(\mathrm{a})$ is for axisymmetric loadings $\left(\Sigma_{y y}=\Sigma_{z z}\right)$ and Figure $7(\mathrm{~b})$ for non-axisymmetric ones.

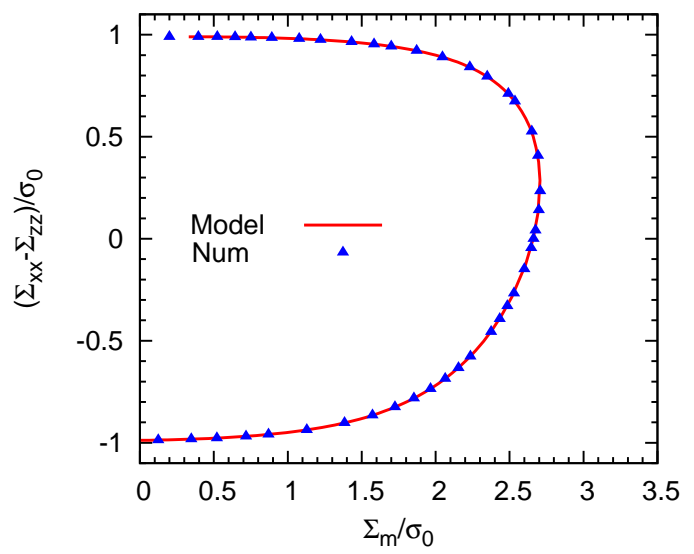

(a) $\Sigma_{y y}=\Sigma_{z z} \neq \Sigma_{x x}$

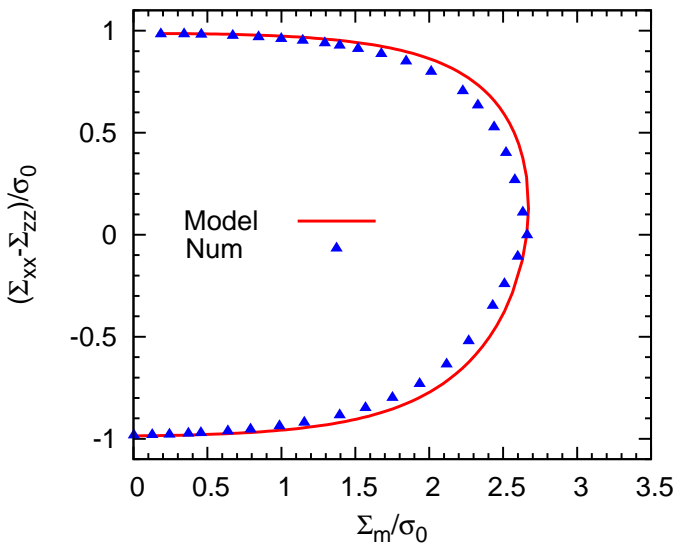

(b) $\Sigma_{x x}=\Sigma_{y y} \neq \Sigma_{z z}$

Fig. 7. Traces in two planes of the yield locus for a circular cylindrical void - $(a, b, c)=(+\infty, 5,5)$, $f=0.01$ - Numerical results and model predictions (Madou-Leblond)

It has been noted by Madou and Leblond (2012b) that for the geometry considered and axisymmetric loadings, their yield surface strictly coincides with those of Gurson and GLD, which are known to be exact in this specific case. Therefore the perfect coincidence of the numerical and theoretical yield loci in Figure $7(\mathrm{a})$ is a new illustration of the accuracy of the finite element results. On the other hand a slight discrepancy between the finite element results and the model predictions may be observed in Figure 7(b), for 
non-axisymmetric loadings. This discrepancy essentially arises from presence of a slight corner on the numerical yield locus on the hydrostatic axis $\left(\Sigma_{x x}-\Sigma_{z z}\right) / \sigma_{0}=0$, which obviously cannot be reproduced by Madou-Leblond's approximate yield function, owing to its $\mathcal{C}^{\infty}$ regularity. The presence of such a corner was established previously both in the theoretical works of Ponte-Castaneda (2002a,b) and the numerical one of Pastor and Ponte-Castaneda (2002), which thus find a confirmation here.

\subsection{Elliptic cylindrical void}

Figure 8 shows results for an elliptic cylindrical void having $(a, b, c)=(+\infty, 5,1)$ and $f=0.01$. In this case absence of any rotational symmetry of the geometry makes it necessary to plot the traces of the yield locus in three planes instead of just two. The agreement of numerical results and model predictions is acceptable in all cases although slight corners may again be observed near (not exactly on) the hydrostatic axis in Figures $8(\mathrm{a})$ and $8(\mathrm{~b})$.

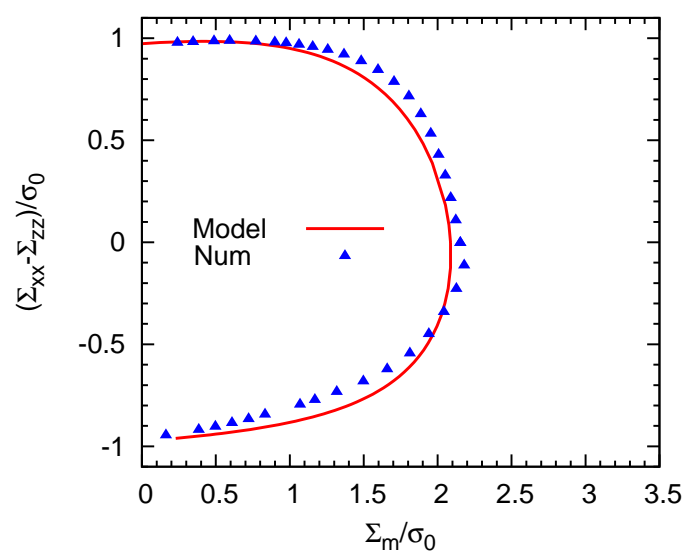

(a) $\Sigma_{x x}=\Sigma_{y y} \neq \Sigma_{z z}$

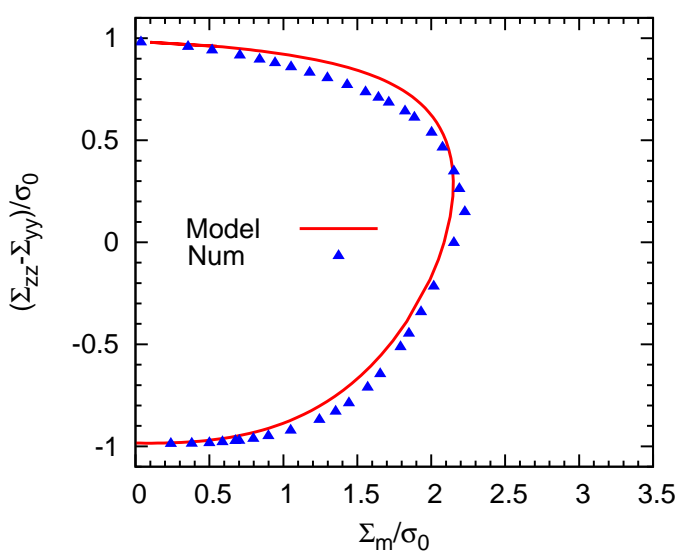

(b) $\Sigma_{x x}=\Sigma_{z z} \neq \Sigma_{y y}$

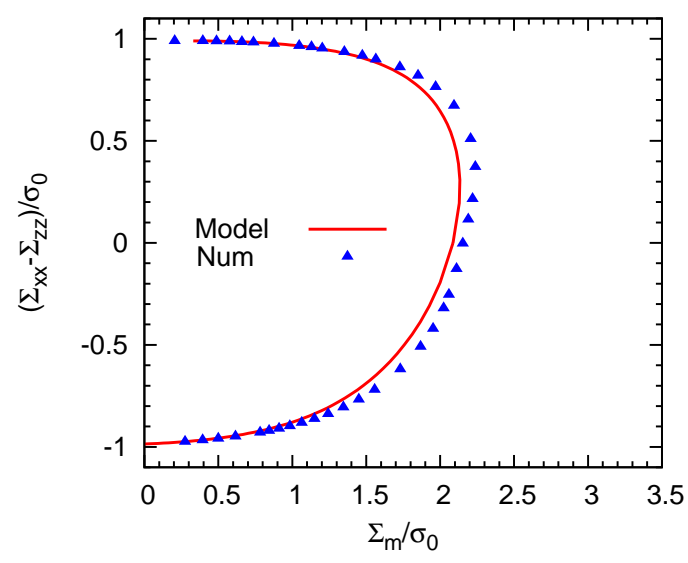

(c) $\Sigma_{y y}=\Sigma_{z z} \neq \Sigma_{x x}$

Fig. 8. Traces in three planes of the yield locus for an elliptic cylindrical void $(a, b, c)=(+\infty, 5,1), f=0.01$ - Numerical results and model predictions (Madou-Leblond) 


\subsection{Circular crack}

Figure 9 is for a circular crack (extreme case of an oblate spheroidal void). Since taking a strictly zero minor axis would raise meshing difficulties and make convergence of the global elastoplastic iterations hazardous, the crack is schematized as a very flat oblate spheroidal void having $(a, b, c)=(5,5,0.2)$ (the minor axis is thus only $1 / 25$ of the major one). Also, the "second porosity" $g$, defined by Madou and Leblond (2012b) as that corresponding to a fictitious spherical void having the same radius, is taken to be 0.14 , in order to allow for comparisons with Gologanu et al. (1994); Gologanu (1997); Gologanu et al. (1997)'s earlier numerical simulations performed for this specific value. Again, Figure 9(a) is for axisymmetric loadings, for which Gologanu et al. (1994); Gologanu (1997); Gologanu et al. (1997)'s earlier results are available and represented, whereas Figure 9(b) is for non-axisymmetric loadings and displays only the new results.

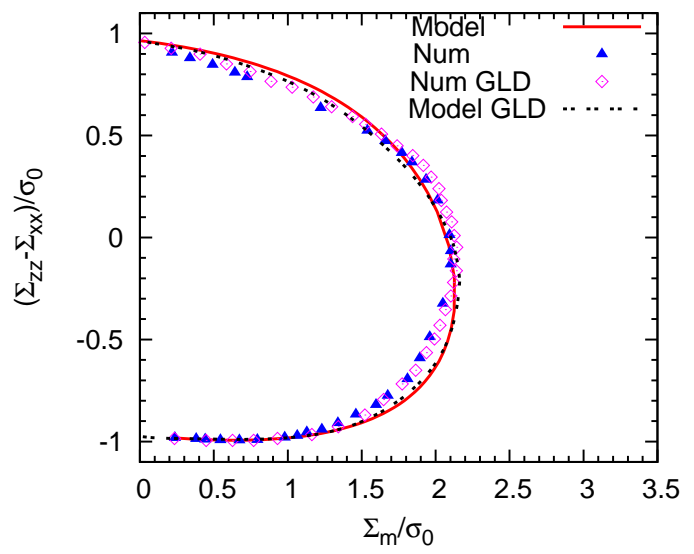

(a) $\Sigma_{x x}=\Sigma_{y y} \neq \Sigma_{z z}$

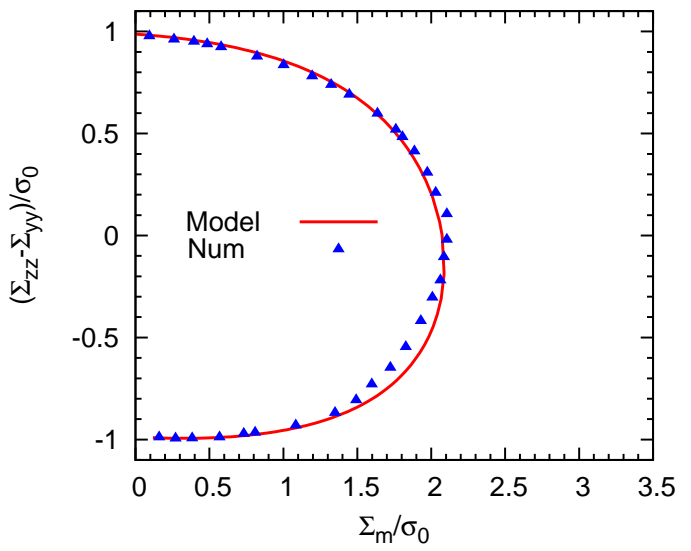

(b) $\Sigma_{x x}=\Sigma_{z z} \neq \Sigma_{y y}$

Fig. 9. Traces in two planes of the yield locus for a circular crack - $(a, b, c)=(5,5,0.2), g=0.14$ - Numerical results and model predictions (Madou-Leblond, GLD)

Again, one may note the quite acceptable agreement of old and new numerical results, in spite of the widely different methods employed, and the very good job made by both the Madou-Leblond and GLD models of reproducing these results.

\subsection{Elliptic crack}

Figure 10 shows the results for an elliptic crack, schematized as a very flat void having $(a, b, c)=(5,2,0.2)$ and $g=0.14$ ( $g$ being defined in this case as the porosity corresponding to a fictitious prolate spheroidal void obtained by rotating the crack about its major axis, see Madou and Leblond (2012b)). Like in the case of an elliptic cylindrical void, absence of any rotational symmetry makes it necessary to plot the traces of the yield locus in three distinct planes. The agreement of finite element results and model predictions is again good, although slightly less satisfactory than for the geometries envisaged so far in the planes $\Sigma_{x x}=\Sigma_{y y}$ and $\Sigma_{x x}=\Sigma_{z z}$. 


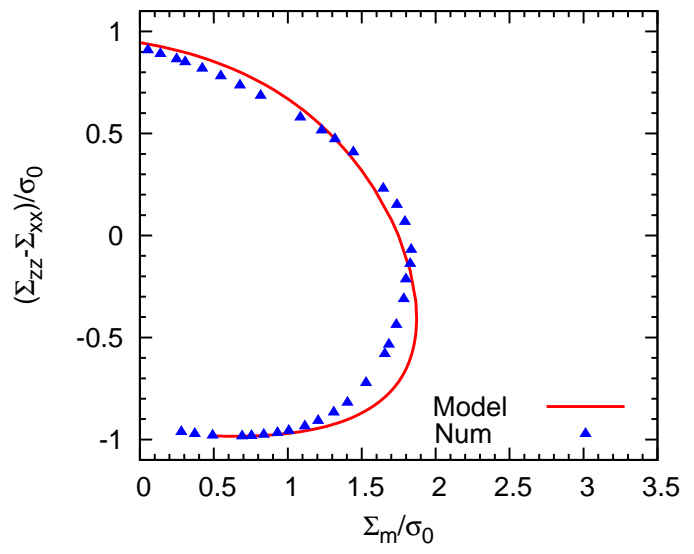

(a) $\Sigma_{x x}=\Sigma_{y y} \neq \Sigma_{z z}$

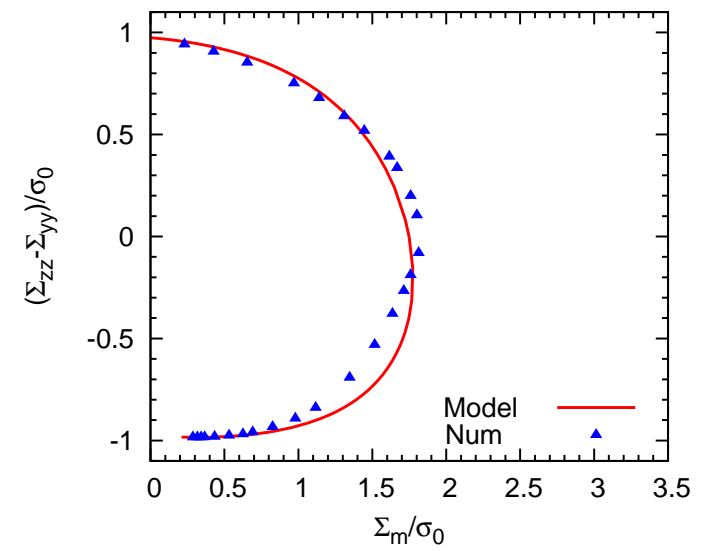

(b) $\Sigma_{x x}=\Sigma_{z z} \neq \Sigma_{y y}$

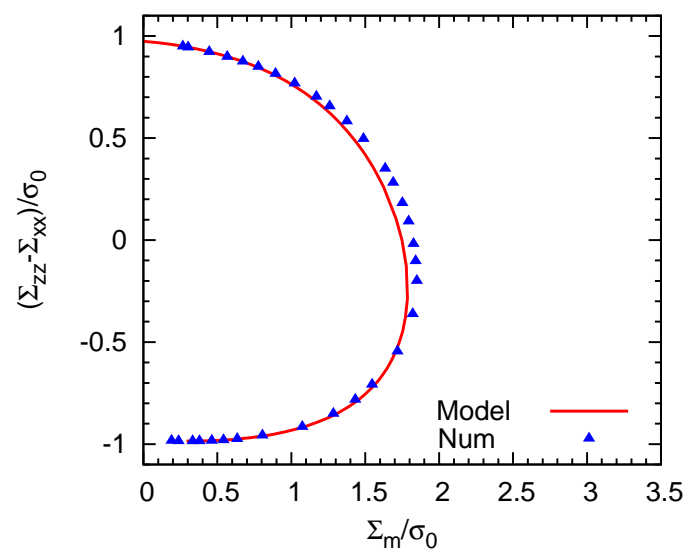

(c) $\Sigma_{y y}=\Sigma_{z z} \neq \Sigma_{x x}$

Fig. 10. Traces in three planes of the yield locus for an elliptic crack - $(a, b, c)=(5,2,0.2)$, $g=0.14$ - Numerical results and model predictions (Madou-Leblond)

\subsection{General ellipsoidal voids}

Figures 11 and 12 finally provide results for two general ellipsoidal voids having $(a, b, c)=$ $(10,5,1)$ and $(a, b, c)=(10,2,1)$ respectively, and $f=0.01$. The traces of the yield loci are again represented in three planes. The agreement between numerical results and model predictions is once again quite acceptable.

\section{Conclusion}

This paper was devoted to the numerical determination of the overall yield surfaces of a number of ellipsoidal elementary cells containing a confocal ellipsoidal void. The aim was essentially to validate Madou and Leblond (2012a,b)'s recently proposed approximate yield criterion for such cells, but the collection of numerical yield surfaces determined may potentially be used to assist in the definition of any similar criterion for plastic porous 


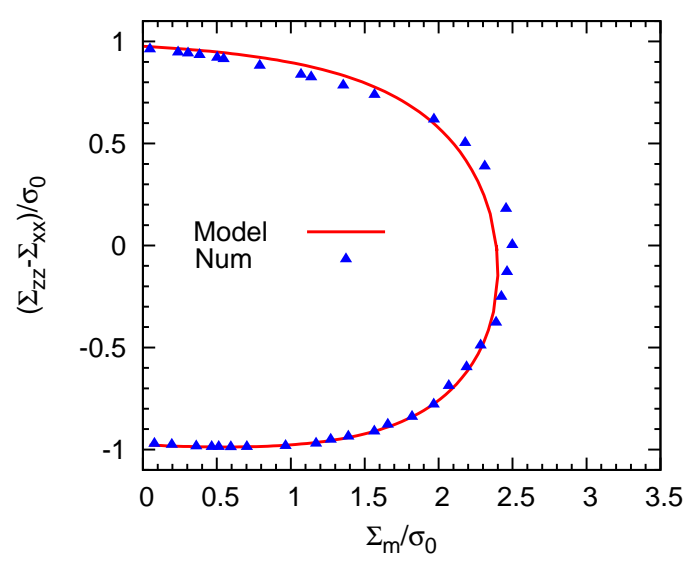

(a) $\Sigma_{x x}=\Sigma_{y y} \neq \Sigma_{z z}$

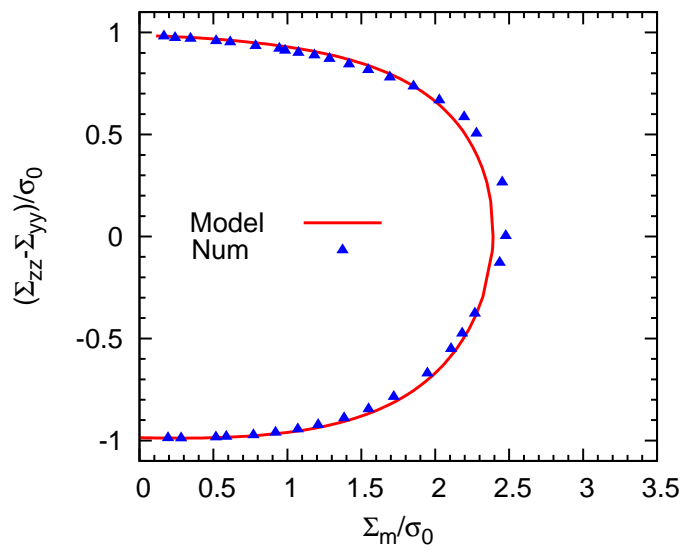

(b) $\Sigma_{x x}=\Sigma_{z z} \neq \Sigma_{y y}$

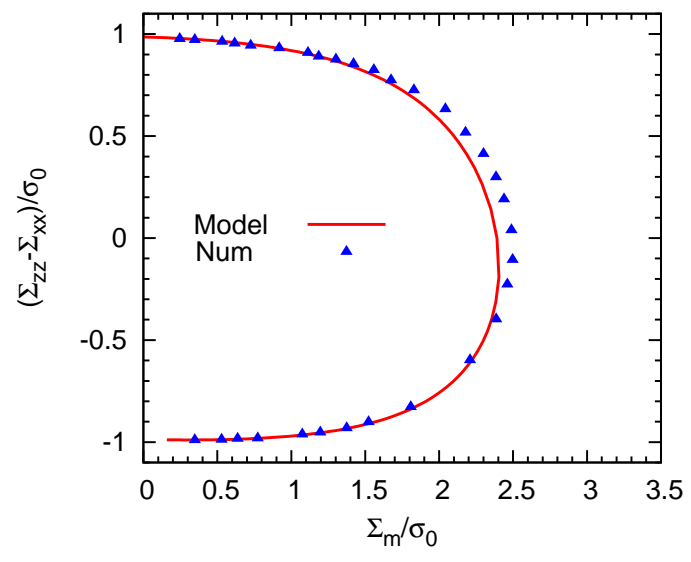

(c) $\Sigma_{y y}=\Sigma_{z z} \neq \Sigma_{x x}$

Fig. 11. Traces in three planes of the yield locus for an ellipsoidal void - $(a, b, c)=(10,5,1)$, $f=0.01$ - Numerical results and model predictions (Madou-Leblond)

materials incorporating void shape effects.

The calculations, based on numerical limit-analysis, were performed using a standard finite element code including elasticity, the equivalence of the time-discretized finite element equations and those of limit-analysis being warranted by use of a single large loading step. The accuracy of the results thus obtained was apparent from comparisons with Gurson (1977)'s exact criterion for a circular cylindrical void subjected to some axisymmetric loading, and with numerical calculations of Gologanu et al. (1993, 1994); Gologanu (1997); Gologanu et al. (1997) for prolate and oblate spheroidal voids.

Yield surfaces were determined for eight different void geometries covering a wide variety of situations: prolate and oblate spheroids, circular and elliptic cylinders, circular and elliptic cracks, and two general ellipsoids. The results obtained evidenced the good general quality of the approximation proposed by Madou and Leblond (2012a,b). They also evidenced the limits of their model in that they revealed, in agreement with previous results of PonteCastaneda (2002a,b) and Pastor and Ponte-Castaneda (2002), the presence of some slight corner on the yield surface for cylindrical voids. Such a corner cannot be reproduced by Madou and Leblond (2012a,b)'s yield function because of its $\mathcal{C}^{\infty}$ regularity. 


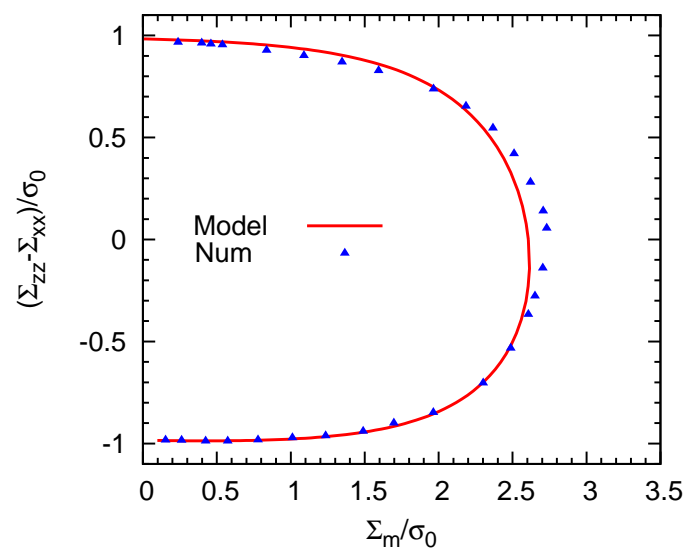

(a) $\Sigma_{x x}=\Sigma_{y y} \neq \Sigma_{z z}$

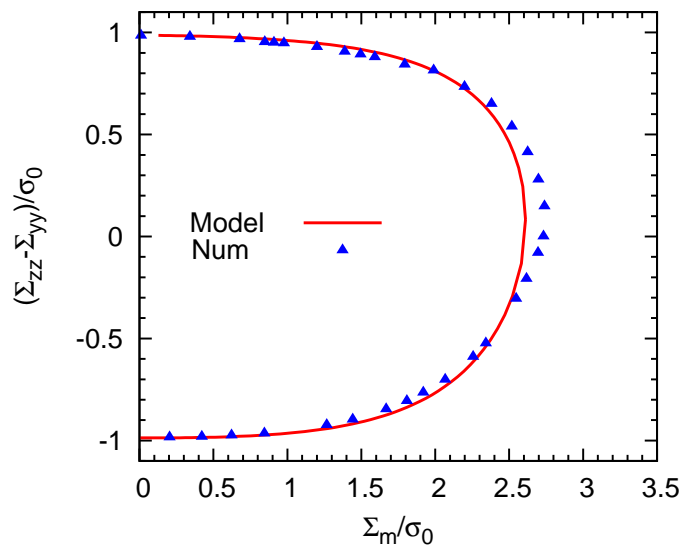

(b) $\Sigma_{x x}=\Sigma_{z z} \neq \Sigma_{y y}$

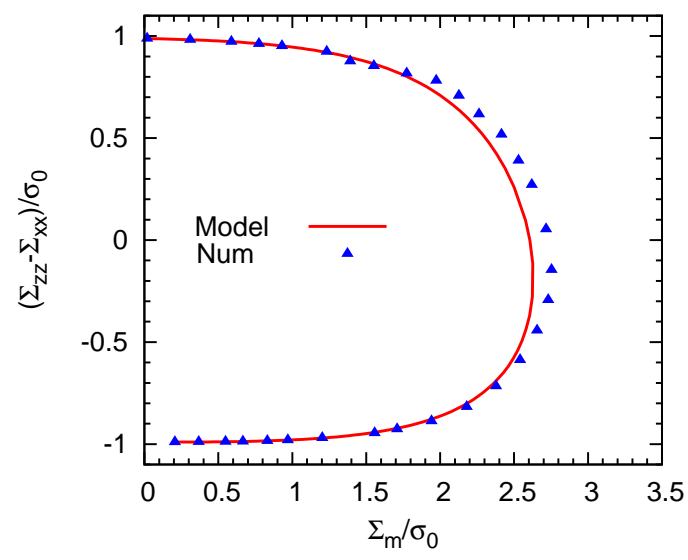

(c) $\Sigma_{y y}=\Sigma_{z z} \neq \Sigma_{x x}$

Fig. 12. Traces in three planes of the yield locus for an ellipsoidal void - $(a, b, c)=(10,2,1)$, $f=0.01$ - Numerical results and model predictions (Madou-Leblond)

Part II will envisage the problem of defining suitable evolution equations for the length and orientation of the axes of the voids, which act as internal parameters in Madou and Leblond $(2012 \mathrm{a}, \mathrm{b})$ 's model and similar ones accounting for void shape.

\section{References}

Agoras M., Ponte Castaneda P. (2013). Iterated linear comparison bounds for viscoplastic porous materials with "ellipsoidal" microstructures. J. Mech. Phys. Solids, 61, 701-725.

Benzerga A., Leblond J.B. (2010). Ductile fracture by void growth to coalescence. Adv. Appl. Mech., 44, 169-305.

Benzerga A., Besson J., Pineau A. (1999). Coalescence-controlled anisotropic ductile fracture. ASME J. Engng. Mater. Technol., 121, 221-229.

Benzerga A., Besson J., Pineau A. (2004). Anisotropic ductile fracture. Part I: experiments. Acta Mater., 52, 4623-4638.

de Buhan P., Maghous S. (1995). A straightforward numerical method for evaluating the ultimate loads of structures. Eur. J. Mech. A/Solids, 14, 309-328. 
Danas K., Ponte-Castaneda P. (2009a). A finite-strain model for anisotropic viscoplastic porous media: I - Theory. Eur. J. Mech. A/Solids, 28, 387-401.

Danas K., Ponte-Castaneda P. (2009b). A finite-strain model for anisotropic viscoplastic porous media: II - Applications. Eur. J. Mech. A/Solids, 28, 402-416.

Gologanu M. (1997). Etude de quelques problèmes de rupture ductile des métaux. Ph.D. Thesis, Université Pierre et Marie Curie (Paris VI) (in French).

Gologanu M., Leblond J.B., Devaux J. (1993). Approximate models for ductile metals containing non-spherical voids - Case of axisymmetric prolate ellipsoidal cavities. $J$. Mech. Phys. Solids, 41, 1723-1754.

Gologanu M., Leblond J.B., Devaux J. (1994). Approximate models for ductile metals containing non-spherical voids - Case of axisymmetric oblate ellipsoidal cavities. ASME J. Engng. Materials Technol., 116, 290-297.

Gologanu M., Leblond J.B., Perrin G., Devaux J. (1997). Recent extensions of Gurson's model for porous ductile metals. In: Continuum Micromechanics, P. Suquet, ed., Springer-Verlag, New-York, pp. 61-130.

Gurson A.L. (1977). Continuum theory of ductile rupture by void nucleation and growth: Part I - Yield criteria and flow rules for porous ductile media. ASME J. Engng. Materials Technol., 99, 2-15.

Hill R. (1967). The essential structure of constitutive laws of metal composites and polycristals. J. Mech. Phys. Solids, 15, 79-95.

Leblond J.B., Gologanu M. (2008). External estimate of the yield surface of an arbitrary ellipsoid containing a confocal void. Comptes-Rendus Mécanique, 336, 813-819.

Lee B., Mear M. (1992). Axisymmetric deformation of power-law solids containing a dilute concentration of aligned spheroidal voids. J. Mech. Phys. Solids, 40, 1805-1836.

Madou K., Leblond J.B. (2012a). A Gurson-type criterion for porous ductile solids containing arbitrary ellipsoidal voids - I: Limit-analysis of some representative cell. J. Mech. Phys. Solids, 60, 1020-1036.

Madou K., Leblond J.B. (2012b). A Gurson-type criterion for porous ductile solids containing arbitrary ellipsoidal voids - II: Determination of yield criterion parameters. $J$. Mech. Phys. Solids, 60, 1037-1058.

Mandel J. (1964). Contribution théorique à l'étude de l'écrouissage et des lois de l'écoulement plastique. In: Proc. 11th Int. Congr. Appl. Mech., Munich, pp. 502-509 (in French).

Michel J.C., Suquet P. (1992). The constitutive law of nonlinear viscous and porous materials. J. Mech. Phys. Solids, 40, 783-812.

Pastor J. (1978). Analyse-limite : détermination de solutions statiques complètes - Application au talus vertical. J.Méc. Appl., 2, 176-196 (in French).

Pastor F., Loute E., Pastor J., Trillat M. (2009). Mixed method and convex optimization for limit-analysis of homogeneous Gurson materials: a kinematical approach. Eur. J. Mech. A/Solids, 28, 25-35.

Pastor F., Ponte-Castaneda P. (2002). Yield criteria for porous media in plane strain: second-order estimates versus numerical results. Comptes-Rendus Mécanique, 330, 741747.

Ponte-Castaneda P. (1991). The effective mechanical properties of nonlinear isotropic materials. J. Mech. Phys. Solids, 39, 45-71.

Ponte-Castaneda P. (2002a). Second-order homogenization estimates for nonlinear composites incorporating field fluctuations. I. Theory. J. Mech. Phys. Solids, 50, 737-757. 
Ponte-Castaneda P. (2002b). Second-order homogenization estimates for nonlinear composites incorporating field fluctuations. II. Applications. J. Mech. Phys. Solids, 50, 759-782.

Ponte-Castaneda P., Zaidman M. (1994). Constitutive models for porous materials with evolving microstructure. J. Mech. Phys. Solids, 42, 1459-1492.

Willis J. (1977). Bounds and self-consistent estimates for the overall moduli of anisotropic composites. J. Mech. Phys. Solids, 25, 185-202.

Willis J. (1991). On methods for bounding the overall properties of nonlinear composites. J. Mech. Phys. Solids, 39, 73-86. 


\section{A Appendix: numerical limit-analysis by the standard finite element method incorporating elasticity}

The equations of limit-analysis are the same as those of a problem of small strain plasticity without elasticity, that is:

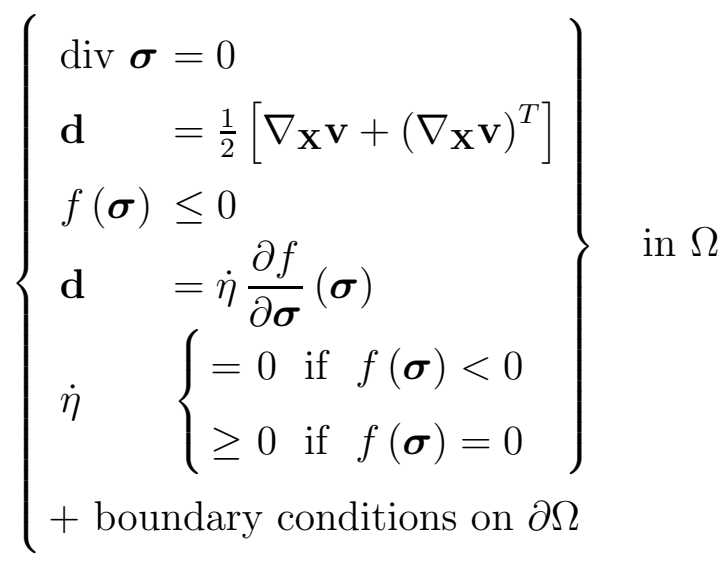

where $\mathbf{X}$ denotes the position-vector in the initial configuration, $\mathbf{v}$ the velocity, $\mathbf{d}$ the strain rate, $\boldsymbol{\sigma}$ the Cauchy stress tensor, $f(\boldsymbol{\sigma})$ the von Mises yield function, $\dot{\eta}$ the plastic multiplier, and $\Omega$ the structure considered.

Now let the elastoplastic problem be solved by the finite element method using an implicit algorithm for the projection of the elastic stress predictor onto the yield locus, with a single large step and no geometry update. Let $\boldsymbol{\sigma}, \mathbf{u}$ and $\boldsymbol{\epsilon}$ denote the final stresses, displacements and strains, respectively. Provided that the load increment is large enough for elastic strains to be generally negligible compared to plastic strains, the equations of the time-discretized problem read

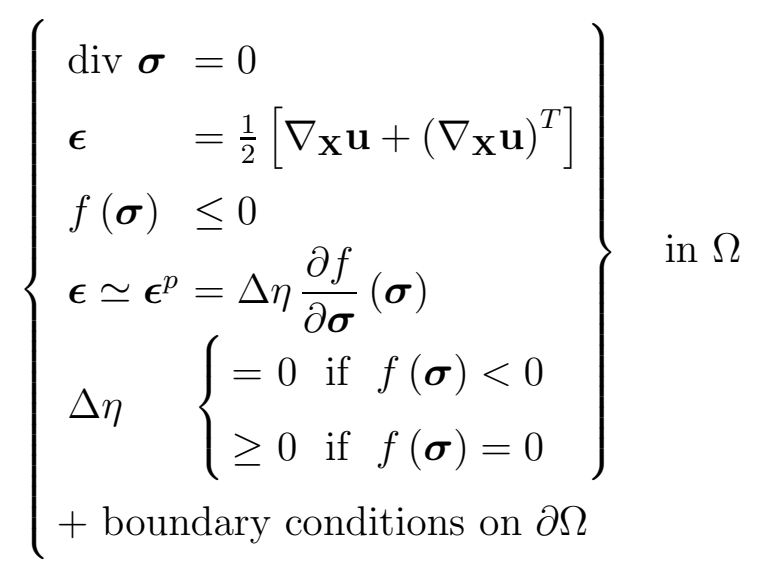

where $\Delta \eta$ denotes the discretized plastic multiplier. The equivalence of systems (A.1) and (A.2) is clear, with the correspondences $\mathbf{v} \leftrightarrow \mathbf{u}, \mathbf{d} \leftrightarrow \boldsymbol{\epsilon}$. (The key point here is that the argument of the derivative $\partial f / \partial \boldsymbol{\sigma}$ in equation (A.2) $)_{4}$ is taken to be $\boldsymbol{\sigma}$ and not $\mathbf{0}$, as required in an implicit algorithm). Thus one may solve a problem of limit-analysis by the standard finite element method, with a single load step sufficiently large to ensure that elastic strains are generally much smaller than plastic ones. 
Under certain conditions, it is even possible to use a smaller load step ensuring only that the limit-load is reached but not that elastic strains are generally negligible. We shall not elaborate on this very useful feature here and just refer the interested reader to (Madou and Leblond, 2012b). 